\title{
Salud neurológica en tiempos de COVID
}

\author{
Neurologic healthcare during COVID emergency \\ Mario Cornejo-Olivas ${ }^{1,2}$, Nilton Custodio ${ }^{3}$, Pilar Mazzetti ${ }^{1,4,5}$
}

La salud neurológica implica una atención multidisciplinaria diferenciada por niveles de atención, la Organización Mundial de la Salud, define a la salud como el estado de completo bienestar físico, mental y social, y no solo la ausencia de afecciones o enfermedades (1). La salud neurológica, involucra entonces no solamente la ausencia de enfermedades que afecten el sistema nervioso, sino también de bienestar del individuo preservando su capacidad de relación con su entorno. En este contexto, los equipos de salud en atención primaria, los especialistas en neurología y los diferentes equipos multidisciplinarios en salud son los responsables de brindan atención sanitaria de las enfermedades neurológicas.

La oferta de atención neurológica actual es creciente, pero aún insuficiente en el país. Según los registros del Colegio Médico del Perú, contamos con alrededor de 700 neurólogos colegiados en todo el país, y 350 de ellos afiliados a la Sociedad Peruana de Neurología (2). Un número no solo insuficiente para satisfacer la demanda de atención de una población de más de 32 millones de habitantes, sino también muy desproporcionado entre regiones y altamente centralizado en Lima, la ciudad capital. La oferta de atención neurológica está segmentada, como en las demás especialidades, entre establecimientos del Ministerio de Salud (MINSA), EsSalud, Fuerzas Armadas y Policiales, así como del sector privado. Los hospitales nacionales y regionales cuentan con unidades, servicios o departamentos de neurología, que van especializándose en áreas específicas como enfermedades cerebrovasculares, epilepsia, neurofisiología, neurología de la conducta, neurogenética entre otras.

La pandemia COVID-19 ha motivado drásticos cambios en las estrategias de atención neurológica en el Perú. Desde el primer caso de COVID en el Perú el 6 de marzo de 2020 y la posterior declaración de emergencia sanitaria, se contabilizaron más de 300000 personas infectadas por la COVID-19 y más de 10000 fallecidos en más de 100 días de pandemia (3). Los profesionales de la salud con comorbilidad de riesgo para COVID-19 se mantuvieron en aislamiento domiciliario; en tanto que los profesionales sin factores de riesgo significativos son movilizados progresivamente hacia a la atención creciente de COVID-19. Estas limitaciones en la atención ambulatoria en neurología en los sistemas públicos de salud del país significaron la cancelación de hasta el $80 \%$ de atenciones ambulatorias programadas en centros especializados en neurociencias y hospitales en el país. Esta situación nos obliga a implementar cambios e innovaciones en la atención sanitaria de enfermedades neurológicas, que contemplen medidas como distanciamiento físico y de bioseguridad incorporando modalidades de atención virtuales basadas en telemedicina.

La COVID-19 es una enfermedad multisistémica de patogenia aun poco conocida, que afecta el sistema nervioso. En los últimos 4 meses, publicaciones diversas en China y Europa han descrito entre 45 y 84\% de casos de COVID-19 con algún tipo de afección neurológica. Los síndromes descritos incluyen trastornos del sistema nervioso central como las encefalopatías, encefalitis, ictus y la epilepsia secundaria; así como afecciones periféricas, como las neuropatías

Centro de Investigación Básica en Neurogenética, Instituto Nacional de Ciencias Neurológicas. Lima, Perú.

Centro de Salud global, Universidad Peruana Cayetano Heredia. Lima, Perú.

Servicio de Neurología, Instituto Peruano de Neurociencias. Lima, Perú.

Facultad de Medicina, Universidad Nacional Mayor de San Marcos, Lima, Perú.

5 Comando de Operaciones COVID-19, Ministerio de Salud. Lima, Perú. 
craneales, el síndrome de Guillain Barré, polineuropatía del enfermo crítico entre otros(4). La evidencia disponible, aunque es aún insuficiente, sugiere mecanismos de invasión directa del SARS-COV-2 y activación de cascadas de respuesta inflamatoria y autoinmunidad (5). Asimismo, muchos pacientes con enfermedades neurológicas crónicas han experimentado aparición o exacerbación de sus síntomas. Este incremento de casos se ve facilitado por medidas gubernamentales contradictorias y muchas veces tardías, bajo cumplimiento de las medidas de distanciamiento físico y la cuarentena, la informalidad; pero fundamentalmente por la falta de controles neurológicos periódicos con la consecuente falta de sus prescripciones médicas.

La teleconsulta se ha convertido en una alternativa innovadora y audaz para atender pacientes con enfermedades neurológicas. Los documentos técnicos que normal la telesalud en el Perú (6), y las recomendaciones para la atención por teleneurología de la Academia Americana de Neurología (7) destacan la necesidad de proveer identificación verificada y el consentimiento verbal del paciente antes de iniciar la entrevista. Asimismo, se recomienda que la entrevista no será grabada para proteger la información confidencial de los pacientes. Esta modalidad de consulta permitiría realizar una adecuada anamnesis y la mayor parte del examen neurológico y cognitivo; aunque hay limitaciones para lograr apreciación del tono muscular, nervios craneales, los reflejos osteotendinosos y patológicos. La evaluación de movimientos involuntarios en pacientes con enfermedades neurodegenerativas dependerá de la velocidad de transmisión de datos y el ancho de banda de los servicios del internet, del proveedor de la plataforma virtual y del dispositivo. Estas limitaciones podrían superarse con una primera evaluación presencial.

A nivel mundial, las atenciones neurológicas están incorporando diversas estrategias para disminuir riesgos sin afectar la calidad de la atención. En varios países del mundo se implementan diversas estrategias de atención pre hospitalaria, aplicación de telemedicina en los servicios de emergencia, discusiones de casos de forma remota, aplicación de pautas para el seguimiento ambulatorio de los casos de alta y recomendaciones para atenciones básicas de tele-rehabilitación, así como atención ambulatoria en casos de ictus leve y ataques isquémicos transitorios (8). Recientemente, la American Heart Association / American Stroke Association (AHA/ASA), recomendó que los neurólogos vasculares se adhieran a las directrices de tratamiento de los pacientes para garantizar una atención adecuada a las enfermedades cerebrovasculares durante la pandemia. Se propone reducir el número de miembros de los equipos de respuesta de pacientes con ictus en emergencia, reduciendo el uso de los ya escasos equipos de protección personal. Asimismo, se promueve el uso de la videoconferencia interactiva para la atención de ictus agudos, entre otras estrategias (9). Las asociaciones de neuropsicólogos y grupos de especialistas en deterioro cognitivo han modificado sus estrategias para realizar las evaluaciones cognitivas de manera remota. La atención de enfermedades raras, esta organizando programas de atención por telemedicina y presencial diferenciados que ofrezcan atención oportuna previniendo complicaciones.

La salud neurológica enfrenta retos diversos y requiere el trabajo conjunto para garantizar la atención de personas con afecciones neurológicas. La llegada de la Pandemia de COVID-19 al Perú es probablemente el reto más grande que enfrentamos como nación; afectando no solo la salud de miles de personas sino golpeando severamente toda actividad productiva y de servicios en el país. El conocimiento y manejo de los nuevos síndromes neurológicos asociados a COVID-19 nos permitirá diagnosticar y tratar cada vez mejor a nuestros pacientes. La neurología peruana asume un reto tremendo, una nueva forma de aproximarnos a la persona afectada y su familia, una forma diferente de entendernos entre nosotros y entender el mundo que ahora tenemos. Las neurociencias se desarrollan en una nueva convivencia donde somos, en esta guerra no convencional, el enemigo y el mejor soldado al mismo tiempo.

\section{REFERENCIAS BIBLIOGRAFICAS}

1. Organización Mundial de la Salud . Constitución de la Organización Mundial de la Salud. Documentos básicos, suplemento de la 45a edición. Ginebra: Organización Mundial de la Salud; 2006. (Citado el 30 de junio del 2020) Disponible en: https://www. who.int/governance/eb/who_constitution_en.pdf
2. Colegio Médico del Perú. Consejo Nacional del Colegio Médico del Perú. Lima; Colegio Médico del Perú (Citado 30 de junio de 2020) Disponible en: https://www.cmp.org.pe/

3. Ministerio de Salud. Covid 19 en el Perú. Lima: Ministerio del Salud; 2020. (Citado el 30 de junio de 2020) Disponible en: https://covid19.minsa.gob. pe/sala_situacional.asp 
4. Ahmad I, Rathore FA. Neurological manifestations and complications of COVID-19: A literature review. J Clin Neurosci. 2020; 77: 8-12.doi: 10.1016/j.jocn.2020.05.017

5. Rodríguez Y, Novelli L, Rojas M, De Santis M, Acosta-Ampudia Y, Monsalve DM, et al. Autoinflammatory and autoimmune conditions at the crossroad of COVID-19. J Autoimmun. 2020; 102506. doi: 10.1016/j.jaut.2020.102506

6. Ministerio de Salud. Resolución Ministerial $\mathrm{N}^{\circ}$ 117-2020-MINSA. Directiva para la Implementación y desarrollo de los servicios de telemedicina síncrona y asíncrona. Lima: Ministerio de Salud; 2020 (Citado el 30 de junio de 2020) Disponible en: https://www. gob.pe/institucion/minsa/normas-legales/462720117-2020-minsa
7. American Academy of Neurology. Telemedicine and COVID-19 [Internet]. 2020. Disponible en: https://www.aan.com/siteassets/home-page/toolsand-resources/practicing-neurologist-administrators/telemedicine-and-remote-care/20telemedicine-and-covid19-v103.pdf

8. Sheth S, Wu TC, Sharrief A, Ankrom C, Grotta J, Fisher M, et al. Early lessons from World War COVID reinventing our stroke systems of care. Stroke. 2020; por publicarse. doi: 10.1161/STROKEAHA.120. 030154

9. AHA/ASA Stroke Council Leadership. Temporary Emergency Guidance to US Stroke Centers During the Coronavirus Disease 2019 (COVID-19) Pandemic: On Behalf of the American Heart Association/American Stroke Association Stroke Council Leadership. Stroke. 2020;51(6):1910-1912. doi: 10.1161/STROKEAHA.120.030023 